\title{
In situ gelling systems based on Pluronic F127/Pluronic F68 formulations for ocular drug delivery
}

Article

Accepted Version

Khateb, K. A., Ozhmukhametova, E. K., Mussin, M. N., Seilkhanov, S. K., Rakhypbekov, T. K., Lau, W. M. and Khutoryanskiy, V. V. (2016) In situ gelling systems based on Pluronic F127/Pluronic F68 formulations for ocular drug delivery. International Journal of Pharmaceutics, 502 (1-2). pp. 70-79. ISSN 0378-5173 doi:

https://doi.org/10.1016/j.ijpharm.2016.02.027 Available at https://centaur.reading.ac.uk/58099/

It is advisable to refer to the publisher's version if you intend to cite from the work. See Guidance on citing.

To link to this article DOI: http://dx.doi.org/10.1016/j.ijpharm.2016.02.027

Publisher: Elsevier

All outputs in CentAUR are protected by Intellectual Property Rights law, including copyright law. Copyright and IPR is retained by the creators or other copyright holders. Terms and conditions for use of this material are defined in the End User Agreement. 


\section{CentAUR}

Central Archive at the University of Reading

Reading's research outputs online 


\section{In situ gelling systems based on Pluronic F127 / Pluronic F68 formulations for ocular drug delivery}

Kosai Al Khateb, ${ }^{1}$ Elvira K. Ozhmukhametova, ${ }^{2}$ Marat N. Mussin, ${ }^{2}$ Serzhan K. Seilkhanov, ${ }^{2}$ Tolebai K. Rakhypbekov, ${ }^{2}$ Wing Man Lau, ${ }^{1}$ V Vitaliy V. Khutoryanskiy ${ }^{1}$ *

${ }^{1}$ Reading School of Pharmacy, University of Reading, Whiteknights, PO Box 224, Reading RG6 6AD, United Kingdom.

${ }^{2}$ Semey State Medical University, 103 Abai Street, Semey 071400, Kazakhstan

Corresponding authors:

1) Dr Wing Man Lau email: w.lau@ @reading.ac.uk (please note Wing Man is the First name, no middle name and surname is Lau)

2) Prof Vitaliy V. Khutoryanskiy email: v.khutoryanskiy@ reading.ac.uk

Please also note that for author Kosai Al Khateb, Kosai is the first name and Al Khateb is the surname. 


\begin{abstract}
This study evaluated the use of Pluronic F127 and Pluronic F68 as excipients for formulating in situ gelling systems for ocular drug delivery. Thermal transitions have been studied in aqueous solutions of Pluronic F127, Pluronic F68 as well as their binary mixtures using differential scanning calorimetry, rheological measurements, and dynamic light scattering. It was established that the formation of transparent gels at physiologically relevant temperatures is observed only in the case of $20 \mathrm{wt} \%$ of Pluronic F127. The addition of Pluronic F68 to Pluronic F127 solutions increases the gelation temperature of binary formulation to above physiological range of temperatures. The biocompatibility evaluation of these formulations using slug mucosa irritation assay and bovine corneal erosion studies revealed that these polymers and their combinations do not cause significant irritation. In vitro drug retention study on glass surfaces and freshly excised bovine cornea showed superior performance of 20 wt \% Pluronic F127 compared to other formulations. In addition, in vivo studies in rabbits demonstrated better retention performance of $20 \mathrm{wt} \%$ Pluronic F127 compared to Pluronic F68. These results confirmed that 20 wt \% Pluronic F127 offers an attractive ocular formulation that can form a transparent gel in situ under physiological conditions with minimal irritation.
\end{abstract}

Keywords: in situ gelling systems, ocular drug delivery, Pluronics, gelation, mucoadhesion 


\section{Introduction}

Drugs are commonly administered topically to treat eye conditions. However, the efficiency of traditional ocular formulations is often sub-optimal. This results in substantial drug loss due to poor cornea permeability, nasolacrimal drainage, tear reflex, blinking, and nonproductive absorption. Typically, ocular drug retention is poor with less than $5 \%$ of the dose reaching the target tissues in the eye (Järvinen, 1995; Hughes, 2005; Ludwig, 2005; Morrison, 2014).

Improving pre-corneal drug retention and enhancing corneal tissue permeability are two main approaches to optimise topical ophthalmic drug delivery. In situ gelling systems can offer improved pre-corneal retention, by converting from a liquid eye drop formulation to a gel upon ocular administration. This results in enhanced drug bioavailability (Agrawal, 2012; Thrimawithana, 2012). Gelation in the pre-corneal environment can be triggered by changes in $\mathrm{pH}$, ionic content or temperature. Weakly cross-linked poly(acrylic acid) derivatives with pH-triggered in situ gelation properties, such as Carbopols ${ }^{\circledR}$, have already been exploited commercially as pharmaceutical excipients. However, due to their anionic nature, Carbopols ${ }^{\circledR}$ are likely to have limited utility for cationic drugs, which may form insoluble complexes leading to phase separation (Nurkeeva, 2002; 2004). Hence, there is a clear need in the development of in situ gelling systems based on non-ionic polymers, where gelling properties will not be affected by the complexation with ionic drugs.

Triblock copolymers of polyethylene glycol- $b$-polypropylene glycol- $b$-polyethyleneglycol, available commercially as Pluronics ${ }^{\circledR}$, are non-ionic, water-soluble materials that have attracted a lot of interest as pharmaceutical excipients. These polymers have an amphiphilic character, exhibit surface active properties and are capable of interacting with hydrophobic surfaces and biological membranes (Batrakova, 2008). In aqueous solutions, Pluronics® can self-assemble to form micelles, which have found applications in the solubilisation of poorlysoluble drugs (Oh, 2004; Sek, 2006; Kadam, 2009). Pluronics ${ }^{\circledR}$ are also known to form gels in situ in response to temperature increase (Pandit, 1998; Ibrahim, 2012; Akash, 2015). Because Pluronics ${ }^{\circledR}$ are transparent, they do not interfere with normal vision and are therefore most suited for applications in ophthalmology. Several ocular formulations comprising combinations of Pluronic F127 and alginate (Lin, 2004), chitosan (Varshosaz, 2008), and cellulose ethers (El-Kamel, 2002) have been reported.

Here, we investigate two Pluronics ${ }^{\circledR}$, i.e. Pluronic F127 (F127) and Pluronic F68 (F68), as excipients to formulate in situ gelling systems for ocular administration. The gelation properties of each polymer and their mixtures were evaluated to establish optimal conditions for forming in situ gelling systems. The irritancy potential and corneal retention of fluorescein sodium formulated using these polymers were also established both in vitro and in vivo. 


\section{Materials and Methods}

\subsection{Materials}

Pluronic F68 (Lutrol ${ }^{\circledR}$ F68, EO $\mathrm{E}_{80} \mathrm{PO}_{27} \mathrm{EO}_{80}$ ) and Pluronic F127 (Lutrol $® \mathrm{~F} 127, \mathrm{EO}_{101} \mathrm{PO}_{56}$ $\mathrm{EO}_{101}$ ) were acquired from BASF, Germany. Optimal cutting temperature compound (OCT), $\mathrm{NaCl}$ and $\mathrm{NaHCO}_{3}$ were purchased from Fisher Scientific, UK. $\mathrm{CaCl}_{2} \cdot 2 \mathrm{H}_{2} \mathrm{O}$ was purchased from BDH Laboratory Supplies, UK. Benzalkonium chloride was purchased from Fluka Chemie GmbH, Germany. Ofloxacin and fluorescein sodium were obtained from SigmaAldrich, UK. Vectashield mounting medium with DAPI was purchased from Vector Laboratories Inc., $U K . \mathrm{T}_{\text {zero }}$ pans and $\mathrm{T}_{\text {zero }}$ hermetic lids for differential scanning calorimetry were purchased from TA Instruments, UK.

\subsection{Preparation of Pluronic formulations}

Pluronic formulations were prepared by dissolving the required amount of Pluronic F127 and/or Pluronic F68 in a cold deionised water, simulated tear fluid (STF) or solution of ofloxacin in STF and stirred overnight at $4{ }^{\circ} \mathrm{C}$. Whilst the concentration of each polymer varied from one formulation to another, the total polymer concentration in each formulation was kept at $20 \mathrm{wt} \%$ (Table S, Supporting Information). After a clear solution was formed, the solutions were stored at room temperature for at least 12 hours before experiments commenced. For formulations containing fluorescein sodium salt or ofloxacin as model drugs, a stock solution containing the model drug was used as a solvent for the polymers.

STF was prepared according to Cho et al (2003). In brief, $6.7 \mathrm{~g} \mathrm{NaCl}, 2.0 \mathrm{~g} \mathrm{NaHCO}_{3}$ and $0.08 \mathrm{~g} \mathrm{CaCl}_{2} \cdot 2 \mathrm{H}_{2} \mathrm{O}$ were dissolved in $1 \mathrm{~L}$ of deionised water and stirred at $40^{\circ} \mathrm{C}$ until a clear solution was obtained. It was kept at ambient temperature until needed.

\subsection{Differential scanning calorimetry}

Pluronic solutions were placed in $\mathrm{T}_{\text {zero }}{ }^{\circledR}$ aluminium pans and hermetically sealed. Differential scanning calorimetry (DSC) was performed using the DSC Q2000 (TA Instruments) at a heating rate of $5^{\circ} \mathrm{C} / \mathrm{min}$ between $5-100^{\circ} \mathrm{C}$ under nitrogen atmosphere. The data were analysed using Universal Analysis 2000 software (TA Instruments version 4.5A). The micelle formation temperature $\left(\mathrm{T}_{\mathrm{m}}\right)$ was determined from the endothermic peak in the recorded DSC thermograms.

\subsection{Rheological measurements}

Two batches of the binary Pluronics formulations were used where one used deionized water as a solvent and the other used STF containing $0.3 \mathrm{w} / \mathrm{v} \%$ ofloxacin as a model drug. All samples were kept at $4{ }^{\circ} \mathrm{C}$ for at least 48 hours to minimise the potential impact of shear history on the rheological behaviour of the tested formulations. The required amount was pipetted out and placed on the pre-cooled peltier plate $\left(5^{\circ} \mathrm{C}\right)$ immediately prior to testing. A 
solvent trap was used to minimise evaporation and to keep a solvent saturated atmosphere surrounding the sample.

The gelation behaviour was investigated by AR2000ex rheometer (TA Instruments) using TA Instruments' TRIOS software (version 2.2.0.1327). A temperature ramp in oscillatory mode was conducted using $40 \mathrm{~mm}$ diameter parallel plate geometry between $5-60^{\circ} \mathrm{C}$ at $5^{\circ} \mathrm{C} / \mathrm{min}$ heating rate. The measurement was performed within the linear viscoelastic region (LVR) at $0.1 \%$ strain and $1 \mathrm{~Hz}$. The temperature at which elasticity modulus $\left(G^{\prime}\right)$ and viscous modulus $(G$ ”) had a crossover was considered to be the system gelation temperature.

\subsection{Dynamic light scattering}

Micelle formation was examined using dynamic light scattering (DLS). Samples were dissolved in deionized water $(5 \mathrm{mg} / \mathrm{mL})$. Their micellation behaviour was studied at 10, 25, and $35^{\circ} \mathrm{C}$ using the Zetasizer Nano-S (Malvern) and analysed using Malvern's Zetasizer software (version 7.10).

\subsection{Slug mucosal irritation test}

Limax flavus slugs were collected locally (Reading, UK) and were kept in flat bottom beakers lined with paper towels soaked with STF for 48 hours prior to experiments. Slug mucosal irritation test was conducted according to an in-house protocol (Khutoryanskaya et al., 2008). Individual slugs were placed on Petri dishes lined with filter paper pre-soaked with one of the formulations. The slugs were exposed to the formulations for 1 hour. Vehicle-only (STF) solution served as a negative control and $1 \mathrm{wt} \%$ benzalkonium chloride solution was used as a positive control. The difference in slug weight before $\left(\mathrm{m}_{\mathrm{b}}\right)$ and after $\left(\mathrm{m}_{\mathrm{a}}\right)$ the exposure was determined. Mucus production (MP) was calculated according to the following formula:

$$
\mathrm{MP}=\left(\mathrm{m}_{\mathrm{b}}-\mathrm{m}_{\mathrm{a}}\right) / \mathrm{m}_{\mathrm{b}} \times 100 \%
$$

\subsection{Bovine cornea erosion test}

Experiments were carried out using fresh bovine eyes acquired from a local abattoir (P.C. Turners, Farnborough, UK). Whole eyes were collected from freshly slaughtered cows and transported in a cold box to the lab to be used immediately.

The testing method described by Mun et al (2014) was used. Each bovine eye was visually checked for corneal damage and placed in an $150 \mathrm{~mL}$ glass beaker. The donor part of a Franz cell was placed on top of the upward-facing cornea and fixed in place with cling film. The beaker was then placed in a $37^{\circ} \mathrm{C}$ water bath for $20 \mathrm{~min}$, after which $1 \mathrm{~mL}$ of the samples was placed in the donor compartment for $1 \mathrm{~h}$. The eye was taken out and washed with STF. The corneal area in contact with the sample plus $2 \mathrm{~mm}$ of surrounding corneal tissue was dissected. The cornea segments were immersed in OCT, frozen using dry ice and stored at $80^{\circ} \mathrm{C}$ until use. 
Corneal cross-sectional specimens of $10 \mu \mathrm{m}$ were prepared for microscopy using a microtome within a Bright cryostat (model OTF). They were stained using Vectashield mounting medium with DAPI. Slides were examined using an AXIOCAM MRm 1.3 MP digital camera mounted on a Zeiss AXIO Imager A1 fluorescent microscope with 50x magnification and a DAPI fluorescence filter was used. Photos were acquired using the AXIO Vision V4.8.2.0 software (Zeiss, Germany).

\subsection{In vitro retention tests}

Retention tests were conducted using bovine cornea as described in Cave et al (2012). Dissection of the bovine cornea took place within 2 hours of eyes delivery, where the whole cornea plus 2-3 mm of attached sclera was removed using a sharp blade. Each cornea was washed with STF, wrapped with cling film to reduce dehydration and stored at $4^{\circ} \mathrm{C}$ to be used on the following day.

Extracted cornea was mounted on a glass slide in an incubator at $37^{\circ} \mathrm{C}$ for $15 \mathrm{~min} .200 \mu \mathrm{L}$ of the polymer solutions containing $1 \mathrm{mg} / \mathrm{mL}$ fluorescein sodium was added on top of the cornea. The slide was returned to the incubator for $2 \mathrm{~min}$. A number of consecutive washes with $200 \mu \mathrm{L}$ STF were then performed. After sample administration and following each wash, a photo was taken using Leica DFC3000 G digital camera attached to a Leica MZ10 F stereo-microscope (Leica Microsystems, Germany) fitted with a fluorescein filter (ET GFP) and Leica Application Suit (LAS) software (version 4.4.0, build 454). Images were analysed using ImageJ (version 1.46r). Similar steps were taken to examine the retention of samples placed directly on glass slides without corneal tissue. Results were normalised using the photo taken immediately after each formulation administration instillation (prior to first wash).

\subsection{In vivo experiments}

Ocular administration experiments with the polymer solutions with fluorescein sodium were conducted in vivo on chinchilla rabbits $(2.5-3.0 \mathrm{~kg}, \mathrm{n}=4)$. These experiments were approved by Semey State Medical University ethics committee and were conducted following the ARVO Statement for the Use of Animals in Ophthalmic and Visual Research. Prior to experiments, rabbits were housed in standard cages and allowed free access to food and water.

During the experiments, rabbits were placed in restraining boxes, where their eyes and eyelid movements were not restricted. Aqueous solutions of fluorescein sodium ( $70 \mu \mathrm{L}$ at 1 $\mathrm{mg} / \mathrm{mL}$ ) in $20 \mathrm{w} / \mathrm{v} \%$ Pluronic F-127 or Pluronic F-68 were administered on the rabbit's right cornea. Swabs of tear fluid were carefully taken from the lower part of rabbit's eye at 0,5 , 10, 15, 20, 25, 30, 40, 50 and 60 min with cotton buds. These cotton buds were weighed before and after sampling to determine the weight of tear fluid sampled. The cotton buds were then immersed in $2 \mathrm{~mL}$ of 90 vol. \% ethanol (Romat Ltd, Kazakhstan) for 1 hour to extract fluorescein. The extracts were centrifuged at $8000 \mathrm{rpm}$ for $10 \mathrm{~min}$, following which the absorbance of the supernatant was measured at $\lambda=490 \mathrm{~nm}$ with a PD-303S 
spectrophotometer (Apel Co Ltd, Japan). The concentration of fluorescein in the tear fluid was calculated using a calibration curve.

In addition, digital images were taken at each sampling point with an iSlim 2020AF camera (KYE Systems Corp., Taiwan). A weak UV light from an L89AJ keychain ultraviolet torch (Maplin Electronics, UK) was shone into the eye to improve the detection of fluorescein sodium, which under these conditions showed green fluorescence. Statistical significance was determined using GraphPad Prims (version 6) using t-test with $p$ value $<0.05$.

\section{Results and Discussion}

\subsection{Micelle formation and gelation}

Triblock copolymers of polyethylene glycol- $b$-polypropylene glycol- $b$-polyethyleneglycol are known to exhibit temperature-induced gel formation in aqueous solutions (Akash, 2015). An increase in temperature results in partial dehydration of their macromolecules due to the breaking of some hydrogen bonds with molecules of water. This leads to the formation of micellar structures with the more hydrophobic polypropylene oxide (PPO) block forming the core of micelles. Further increases in temperature cause the micelles to rearrange into a gel structure. This sol-gel phase transition is a reversible process that occurs at a temperature dependent on the chemical structure of the triblock copolymer (i.e. type of Pluronic $®$ and its concentration in solution).

Differential scanning calorimetry (DSC) is a powerful technique to look at temperatureinduced transitions happening in solutions of thermoresponsive polymers (Aseyev, 2011). DSC was used in this work to look at the behaviour of individual F127 and F68 in aqueous solutions, as well as their mixtures (Figure 1). $20 \mathrm{wt} \% \mathrm{~F} 127$ solutions showed an endotherm with an onset temperature $\left(\mathrm{T}_{\text {onset }}\right)$ at $13.7^{\circ} \mathrm{C}$, peak at $16.8^{\circ} \mathrm{C}$ and endset temperature $\left(\mathrm{T}_{\text {endset }}\right)$ at $27.0^{\circ} \mathrm{C}$, which is in agreement with the results reported by Pham Trong et al (2008). This endotherm is related to the micelle formation. $T_{\text {onset }}$ is believed to correspond to the beginning of micelle formation, whereas $\mathrm{T}_{\text {endset }}$ shows the completion of micellisation process (Zhang, 2013). F68 solutions showed a different thermal behaviour from F127: the endotherm shifted to a higher temperature range with $\mathrm{T}_{\text {onset }}=35.9^{\circ} \mathrm{C}$, peak at $45.40{ }^{\circ} \mathrm{C}$ and $\mathrm{T}_{\text {endset }}=60.8{ }^{\circ} \mathrm{C}$. The micellisation peak of F68 was substantially smaller compared to F127. DSC thermograms of F127/F68 mixtures containing 2-10 wt \% F68 showed a single endotherm at a lower temperature range $\left(\mathrm{T}_{\text {onset }}=14.3-15.0^{\circ} \mathrm{C}, \mathrm{T}_{\text {peak }}=17.4-17.6^{\circ} \mathrm{C}\right.$ and $\mathrm{T}_{\text {endset }}=30.1-$ $32.4{ }^{\circ} \mathrm{C}$ ). These results are broadly in agreement with the data published by Zhang et al (2013), who reported that diluting down a $20 \mathrm{wt} \%$ solution of F127 increased its critical micelle temperature (CMT), whereas F127/F68 mixtures with varying F127 concentrations but a constant total concentration of F127 and F68 (20 wt \%) exhibited a relatively stable micellisation temperature. Furthermore, their Micro-DSC data provided some evidence to the formation of separate micelles for each Pluronic copolymer rather than hybrid ones within the binary systems. Our results (Table 1) show a similar trend to those reported by Zhang et al 
(2013). Increasing F68 content at the expense of F127 while keeping a fixed total system concentration at $20 \mathrm{wt} \%$ resulted in a relatively constant micellisation temperature $\left(\mathrm{T}_{\mathrm{m}}\right)$. However, the recorded $\mathrm{T}_{\mathrm{m}}$ are $4-5^{\circ} \mathrm{C}$ higher than previously reported (Zhang, 2013). This could be related to variations in molecular weight and relative content of polypropylene oxide (PPO) and polyethylene oxide (PEO) blocks of Pluronic F127 that was used in both sets of experiments. Such variations, mainly PPO content, directly affect the thermal behaviour of dilute Pluronic polymers solutions (Beezer, 1994). In addition, the shorter PPO chains of F127 (56 units) in the current work are associated with higher $\mathrm{T}_{\mathrm{m}}$ compared to Zhang et al (2013) samples (69 units).

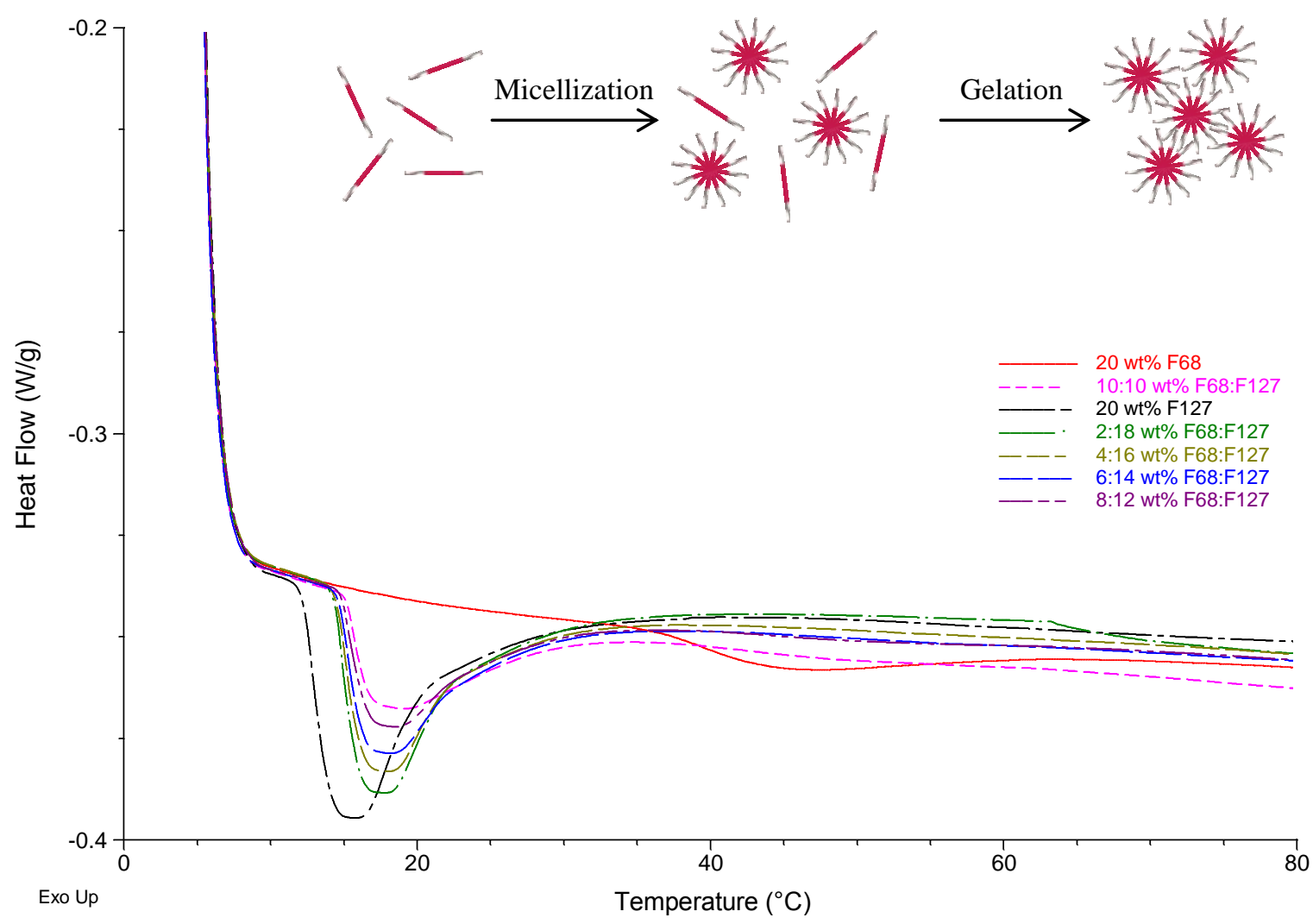

Figure 1. DSC thermograms of F68, F127 and their mixtures. All binary systems show relatively similar thermal changes which are related to micelles formation. Inset: schematic representation of the micellation/gelation processes in Pluronic F127 single system. 
Table 1. Micellization and gelation temperatures in F68/F127 individual polymer solutions and binary mixtures. For micellization temperature $\left(T_{m}\right)$, formulations were prepared in deionised water. For gelation temperature (Tg), two sets were prepared, one using deionised water and the other using simulated tear fluid containing $30 \mathrm{mg} / \mathrm{mL}$ of ofloxacin as a model drug (mean \pm s.d., $n=3$ ).

\begin{tabular}{|c|c|c|c|}
\hline $\begin{array}{c}\text { F68:F127 } \\
(\mathbf{\%} \text { w/v) }\end{array}$ & $\begin{array}{c}\text { Micellation Temperature } \\
\left(\mathbf{T}_{\mathbf{m}}\right) \text { of samples in } \\
\text { deionised water }\left({ }^{\circ} \mathbf{C}\right)\end{array}$ & $\begin{array}{c}\text { Gelation temperature } \\
(\mathbf{T g}) \text { of samples in } \\
\text { deionised water }\left({ }^{\circ} \mathbf{C}\right)\end{array}$ & $\begin{array}{c}\text { Gelation temperature } \\
(\mathbf{T g}) \text { of samples in STF } \\
\text { ofloxacin solution }\left({ }^{\circ} \mathbf{C}\right)\end{array}$ \\
\hline $\mathbf{0 : 2 0} *$ & 16.8 & $28.1 \pm 0.5$ & $27.2 \pm 0.4$ \\
\hline $\mathbf{2 : 1 8}$ & 17.4 & $38.6 \pm 1.8$ & $40.5 \pm 1.7$ \\
\hline $\mathbf{4 : 1 6}$ & 17.3 & $48.0 \pm 1.9$ & $48.1 \pm 1.6$ \\
\hline $\mathbf{6 : 1 4}$ & 17.5 & $62.8 \pm 4.8$ & $61.2 \pm 1.8$ \\
\hline $\mathbf{8 : 1 2}$ & 17.6 & $65.9 \pm 3.3$ & $68.1 \pm 4.5$ \\
\hline $\mathbf{1 0 : 1 0}$ & 17.6 & $71.4 \pm 4.0$ & $69.5 \pm 2.8$ \\
\hline $\mathbf{2 0 : 0} *$ & 45.7 & $74.7 \pm 1.1$ & $75.6 \pm 4.3$ \\
\hline
\end{tabular}

*Note that these entries correspond to $20 \%$ w/v solutions of individual F127 and F68, respectively

Furthermore, Micro-DSC results reported by Zhang et al (2013) were using heating rate of $0.1^{\circ} \mathrm{C} / \mathrm{min}$, while our study was performed at $5^{\circ} \mathrm{C} / \mathrm{min}$. Such significant change in heat flow could have an effect on the recorded DSC thermogram, as higher heating rate (faster heat flow) can introduce latency in the DSC sensors ability to detect the start of thermal changes caused by micellation. This may potentially lead to a delay in the detection process, thus higher recorded values. Another factor is the preparation method, as polymers in the current work were prepared by stirring the solid Pluronics in the appropriate solvent, while Zhang et al (2013) prepared stock solutions of each Pluronic copolymer prior to binary systems preparation. This may have an effect on the distribution and possibly the aggregation of unimers of Pluronics, which consequently may affect the nature of formed micelles.

According to Zhang et al (2013), when the content of F68 was relatively high in the binary system ( $\geq 10 \mathrm{wt} \%$ ), two endothermic peaks were seen which were thought to correspond to the separate micellation event of F127 and F68. However, these secondary peaks were not detected for any binary solutions in the current work. Nevertheless, the differences in the DSC instrument used in the present work and the more sensitive Micro-DSC used by Zhang et al (2013) may explain the missing F68 peaks.

Rheological measurements provide an excellent opportunity to monitor gelation in solutions. According to Wei et al (2002), a formulation is forming a gel if its elasticity modulus ( $G^{\prime}$ ) and viscous modulus $\left(G^{\prime \prime}\right)$ are frequency-independent, and has a low phase angle $\delta(\tan \delta$ $\left.=G{ }^{\prime \prime} / G^{\prime}\right)$ at all frequencies. Therefore, in the present study, the linear viscoelastic region (LVR) for F127, F68 and their binary mixtures was determined and consequent rheological measurements were conducted at a frequency of $1 \mathrm{~Hz}$. The temperature at which both moduli $G^{\prime}$ and $G$ ' had a crossover was considered as the gelation temperature (Tg) (Figure 2). 


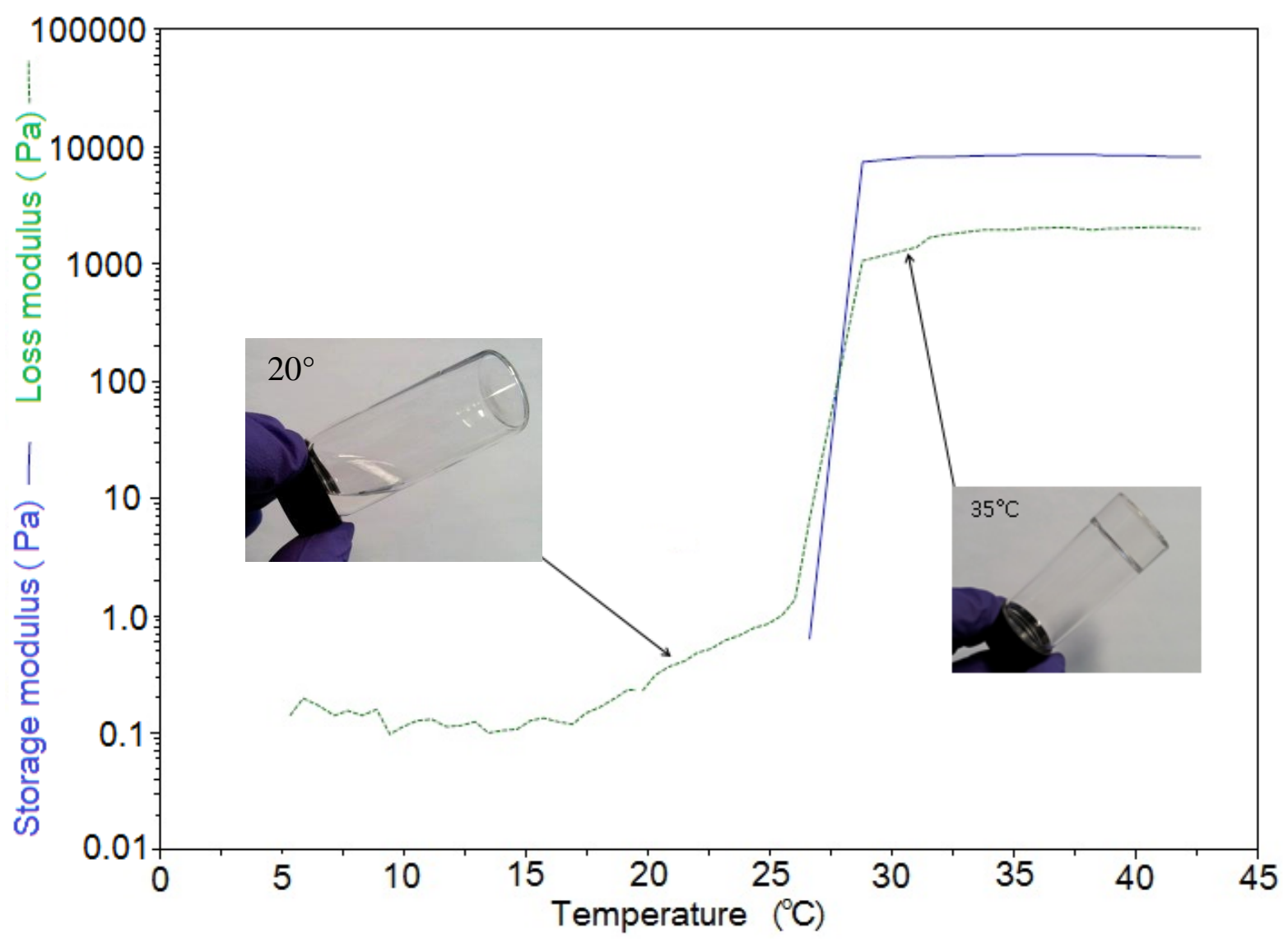

Figure 2. Exemplary rheological profile of $20 \mathrm{wt} \%$ F127. The increase in G' over G" represents an increase in solid over liquid characteristics of the tested sample, which reflects a phase transition into a "gel" structure. A crossover of elastic with loss moduli (G' vs G", respectively) indicates the gelation temperature. Below $25^{\circ} \mathrm{C}, \mathrm{G}$ ' values are very low and close to the detection limits of the rheometer, which is related to the liquid nature of the solution. G' shows a significant increase between $25^{\circ} \mathrm{C}-30^{\circ} \mathrm{C}$, which corresponds to the solgel transition process. Insets: photos showing the phase transition (gelation) of $20 \mathrm{wt} \% \mathrm{~F} 127$ at $20^{\circ} \mathrm{C}$ and $35^{\circ} \mathrm{C}$ using the vial inversion method.

The results (Table 1) show that increasing F68 concentration causes an increase in $\mathrm{Tg}$ for binary systems both dissolved in deionised water and in STF. Zhang et al (2013) results also showed an increasing trend of the gelation temperature by increasing F68 content in the binary system. This can be explained by the reduction in F127 content and thus the decrease in the micellization and consequent rearrangement of Pluronic micelles which is responsible for the gelation process. However, their Tg data were significantly different to our study. These differences can again be attributed to the variations in the molecular weight of tested Pluronic F127 and its PPO content, method of preparation (polymer mixing), use of different rheometer geometries as well as the heating rate which were discussed earlier.

Previous studies have demonstrated the effect of different additives on the micellisation/gelation behaviour of Pluronic F127 (Edsman et al, 1998. Sharma et al, 2004. 
Chung et al, 2006). These changes in behaviour depended mainly on the hydrophobicity of the solutes and the presence of cosolvents (Sharma et al, 2004). In the present work (Table 1), changing the solvent from deionised water to STF with ofloxacin had a minimal effect on the gelation temperature of the binary Pluronic mixture (Figure S1, Supporting information). These results show that the presence of ofloxacin and salts $\left(\mathrm{NaCl}, \mathrm{NaHCO}_{3}\right.$ and $\left.\mathrm{CaCl}_{2} \cdot 2 \mathrm{H}_{2} \mathrm{O}\right)$ in STF had little effect on the rearrangement of Pluronic micelles and, therefore, on the gelation process.

DLS was used to study the behaviour of micelles/aggregates formed due to the thermal response of Pluronics F127, F68 and their binary solutions. The results indicate that for a binary system, increasing the temperature led to an increase in the diameter of micelles formed, as exemplified by the 6:14 wt\% F68:F127 mixture (Figure 3A). On the other hand, at a given temperature, the micellar diameter was not affected by the composition (molar ratio) of either polymer when the total polymer concentration was fixed at $20 \mathrm{wt} \%$, as all tested systems showed similar size distributions at fixed temperature (Figure 3B). This is in agreement with the recorded $\mathrm{T}_{\mathrm{m}}$ (Table 1), whose values are not dependent on the Pluronics molar ratio in the binary system.

In principle, the monodisperse profile (Figure 3) may suggest that a single type (hybrid) of micelles was formed by the F68 and F127 copolymers in their aqueous mixture. On the other hand, Zhang et al (2013) found the evidence on separate micelles formation using SAXS technique as well as their interpretation of Micro-DSC data. Due to the low resolution of DLS instrument used in the current work, further experimentation with more sensitive techniques is required to confirm the types of micelles formed.
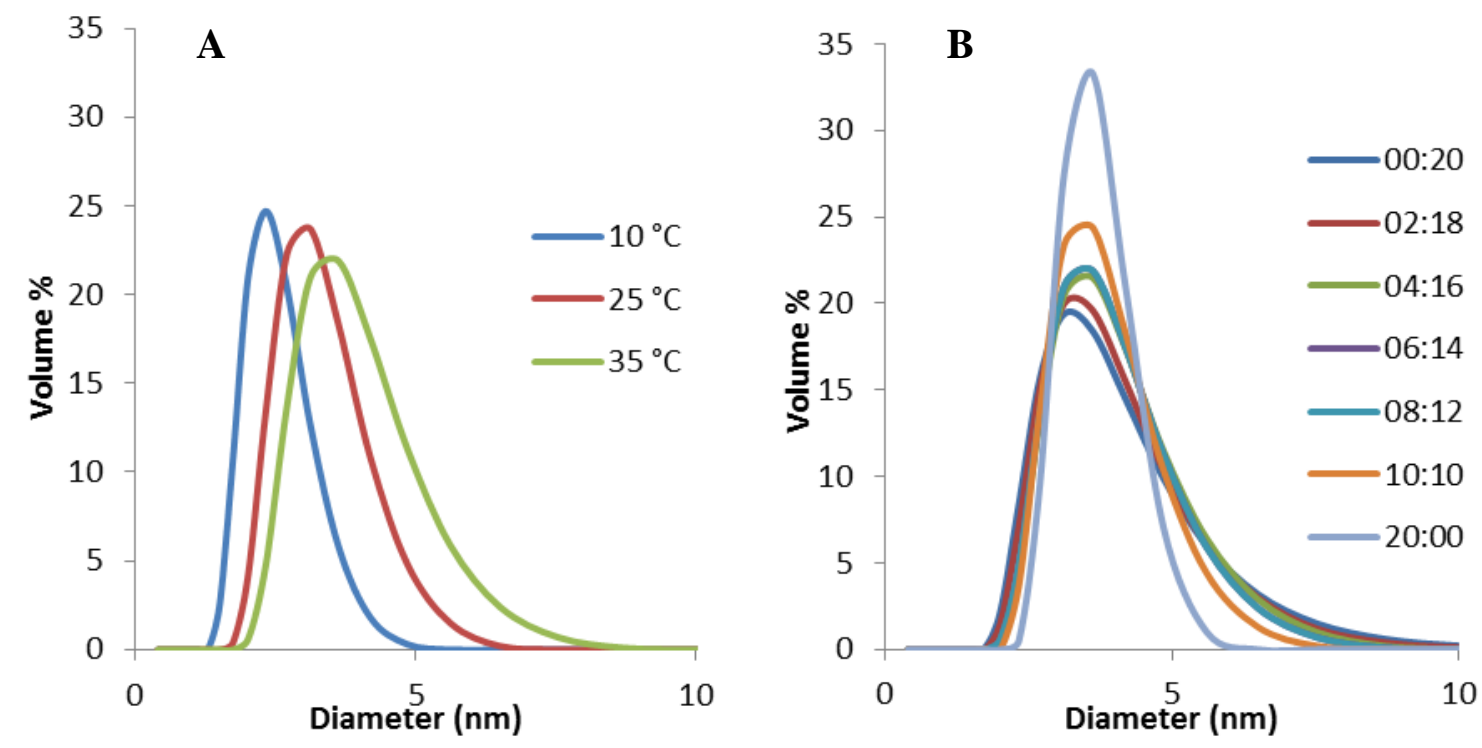

Figure 3. Dynamic light scattering measurements of F68:F127 (6:14 \% w/v) at different temperatures (A), and for different $\mathrm{F} 68: \mathrm{F} 127$ mixtures at $35^{\circ} \mathrm{C}(\mathrm{B})$. All solutions were prepared in deionised water (mean, $n=3$ ). 


\subsection{Toxicity characterisation of Pluronics and their mixtures}

Topical administration of ocular medicines requires the formulations to be non-irritant to the eye. Potential irritation effects of Pluronic formulations on living tissue were studied in Limax flavus slugs. These slugs produce mucus to aid their locomotion, to protect against dehydration, and in response to irritants. Adriaens and Remon have reported this novel slug mucosal irritation test as a method for evaluation of irritancy of various chemicals to mucosal membranes (Adriaens and Remon, 1999). This method involves the measurement of mucus secretion in slugs exposed to different chemicals as a measure of the degree of irritation caused by these agents. This method was validated as an alternative screening test for eye irritation (Adriaens, 2005).

Limax flavus slugs were chosen because they exhibited a greater response (more mucus secretion) to irritants compared to some other species, in addition to their local availability (Khutoryanskaya et al., 2008). We have previously validated the slug mucosal irritation assay against ocular administration of formulations in rabbits (Khutoryanskaya, 2014).

Figure 4 illustrates the effect of various Pluronic formulations on the mucus production in Limax flavus. All Pluronic formulations did not cause a significant increase in mucus production compared to the negative control $(\mathrm{STF}, \mathrm{p}<0.05)$. Indeed, they were significantly $(\mathrm{p}<0.05)$ lower than the positive control $(1 \mathrm{wt} \%$ benzalkonium chloride).
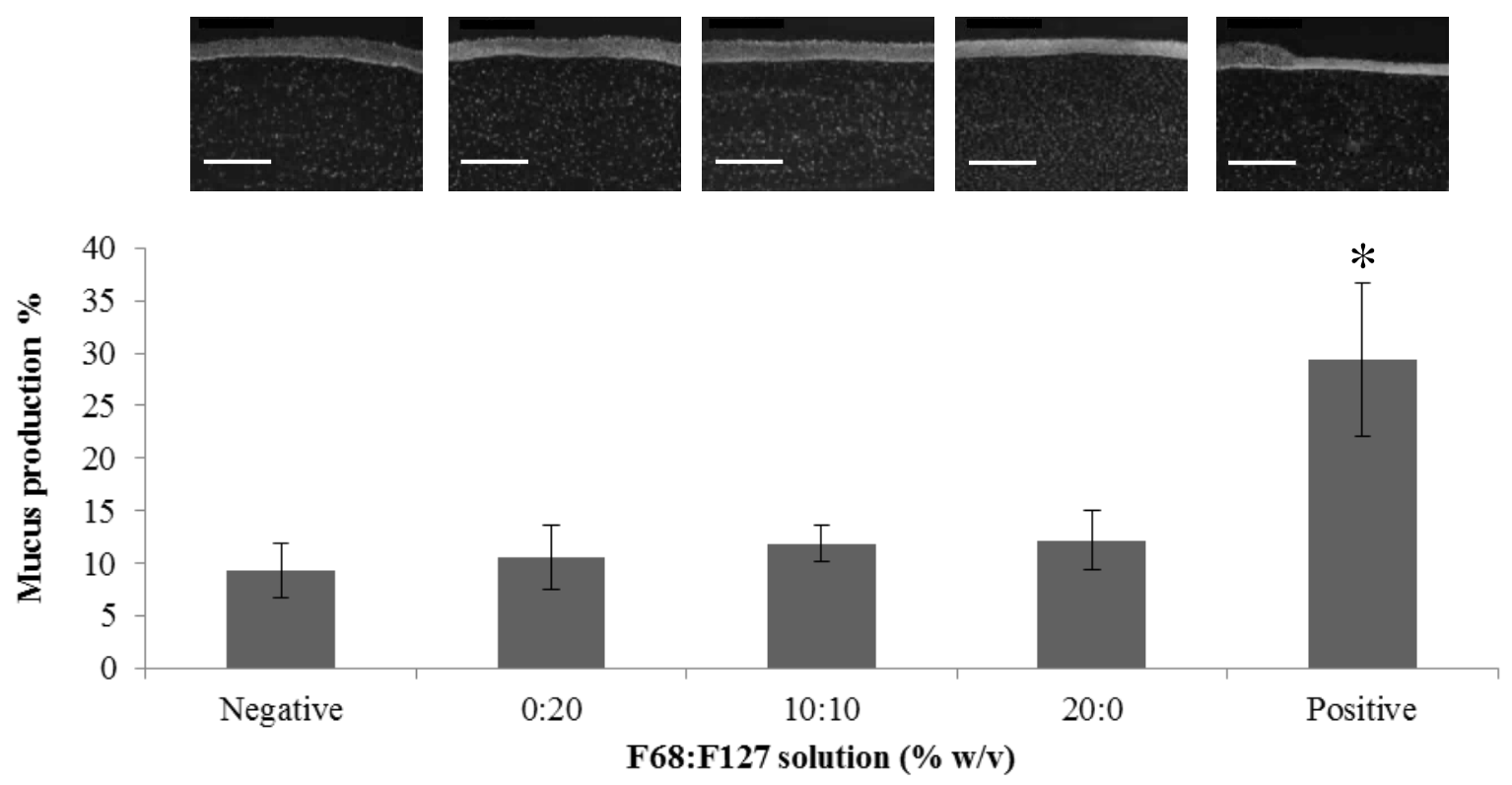

Figure 4. Effect of F68, F127 and their $10 \mathrm{wt} \%-10 \mathrm{wt} \%$ binary mixture on the mucus production in Limax flavus slugs. $1 \mathrm{wt} \%$ benzalkonium chloride in STF and STF only were used as a positive and negative controls, respectively (mean $\pm \mathrm{SD}, \mathrm{n}=3$, * represents $\mathrm{p}<$ 0.05 against negative control). Inset: effect of tested formulations on the erosion of bovine corneal epithelium (scale bar $=0.5 \mathrm{~mm}$ ). The images (left to right) represents the negative 
control, the F68:F127 formulations and the positive control. Photos are shown in black and white to ensure good contrast.

In addition to the slug irritation assay, a novel bovine corneal erosion test was conducted using Pluronic formulations with freshly excised bovine eyes. In this experiment, the whole eye method proposed by Mun et al (2014) was used. Each specimen was examined for variations in epithelial thickness. As the insets in Figure shows, only $1 \%$ benzalkonium chloride caused a substantial change in epithelial thickness, whilst the polymer formulations and the negative control showed no such variations. This change in epithelial thickness was potentially due to the irritation effects of benzalkonium chloride. Furthermore, visual examination of bovine eyes at the end of the experiment ( 1 hour) showed a white discoloration of the corneal tissue where it was in contact with benzalkonium chloride. Such discoloration was not observed with the other samples. Figure S2 (Supporting information) shows more detailed photos from each sample, with changes in thickness of corneal epithelium being obvious in the case of benzalkonium chloride.

The irritation and erosion tests are in agreement with previous reports regarding the safety of Pluronics (Akash, 2015) and also demonstrate the safety of aqueous binary solutions of Pluronic F68 and F127 for ocular applications.

\subsection{In vitro retention of Pluronic formulations on glass surfaces and bovine cornea}

The retention of Pluronic formulations on glass surfaces and freshly excised bovine cornea was initially examined in vitro using our previously reported approach (Irmukhametova 2011, Withers et al, 2013; Cook et al, 2015). Figure 5 shows exemplary fluorescent images and the wash-off profiles for the formulations deposited on the glass surface. A glass slide was used in this case as a non-mucosal surface control to establish whether any retention effects observed could be related simply to the rheological properties of the formulations rather than specific mucoadhesive interactions. The solution of fluorescein sodium in STF did not show any retention on the glass surface and was completely removed from the slide in the first two washes. Very weak retention of fluorescein sodium was also observed in the formulations containing $20 \mathrm{wt} \%$ F68 and F68:F127 mixture (10 wt \% : $10 \mathrm{wt} \%$ ); both formulations were removed from the glass surface during the first two washes with STF. This result was expected as the rheological studies confirmed that these formulations did not form gels at physiological temperature. Free flowing solutions composed of 20 wt \% F68 and F68:F127 mixture have a weak ability to withstand STF fluid wash off effects and do not exhibit sufficient retention on the corneal surface. On the contrary, $20 \mathrm{wt} \% \mathrm{~F} 127$ exhibited excellent resistance to STF and remained on the glass surface even after 7 washes. This can be explained by the sol-gel phase transition of F127 under the experimental conditions. It can be noted that the fluorescence intensity for $20 \mathrm{wt} \%$ F127 showed an increase after the first wash; this is related to the partial sample spreading on the glass surface and broadening of the fluorescent area. The initial fluorescence intensity in these experiments was taken as $100 \%$, 
therefore the broadening of fluorescent area resulting from initial washes leads to $>100 \%$ values.

The formulations exhibited better spreadability on the biological surface compared to the glass surface (Figure 6). Fluorescein sodium solution, 20 wt \% F68 and F68:F127 (10:10\%) mixture showed poor retention. However, traces of fluorescence remained even after 8 consecutive washes. This can potentially be caused by partial absorption/entrapment of fluorescein sodium within the corneal epithelium, or/and by mucoadhesive effects, i.e. specific interactions of Pluronic macromolecules with the ocular surface. Similarly to its retention on glass surfaces, the $20 \mathrm{wt} \% \mathrm{~F} 127$ formulation exhibited excellent retention on corneal surface and resisted 8 washes with STF.

A

a

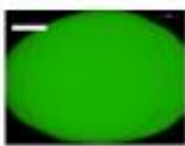

b

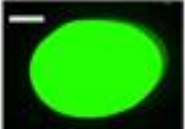

c

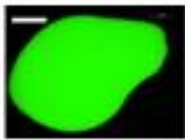

d

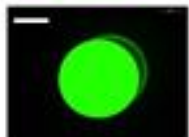

0
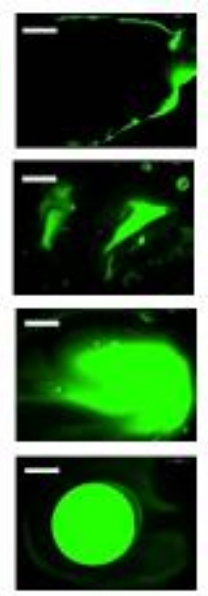

1
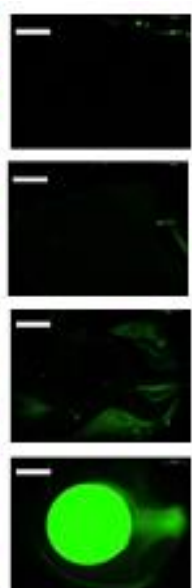

2
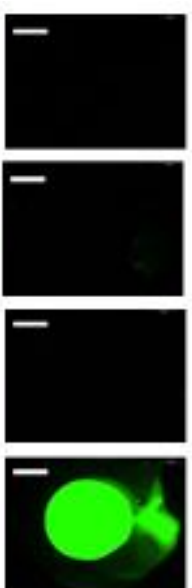

3
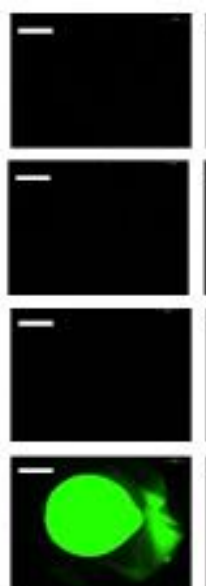

4
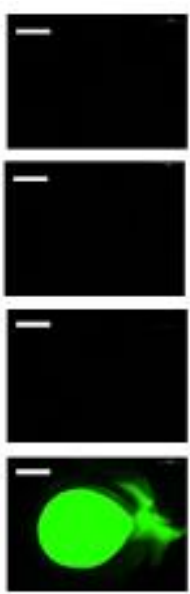

5

Number of washes with STF

B

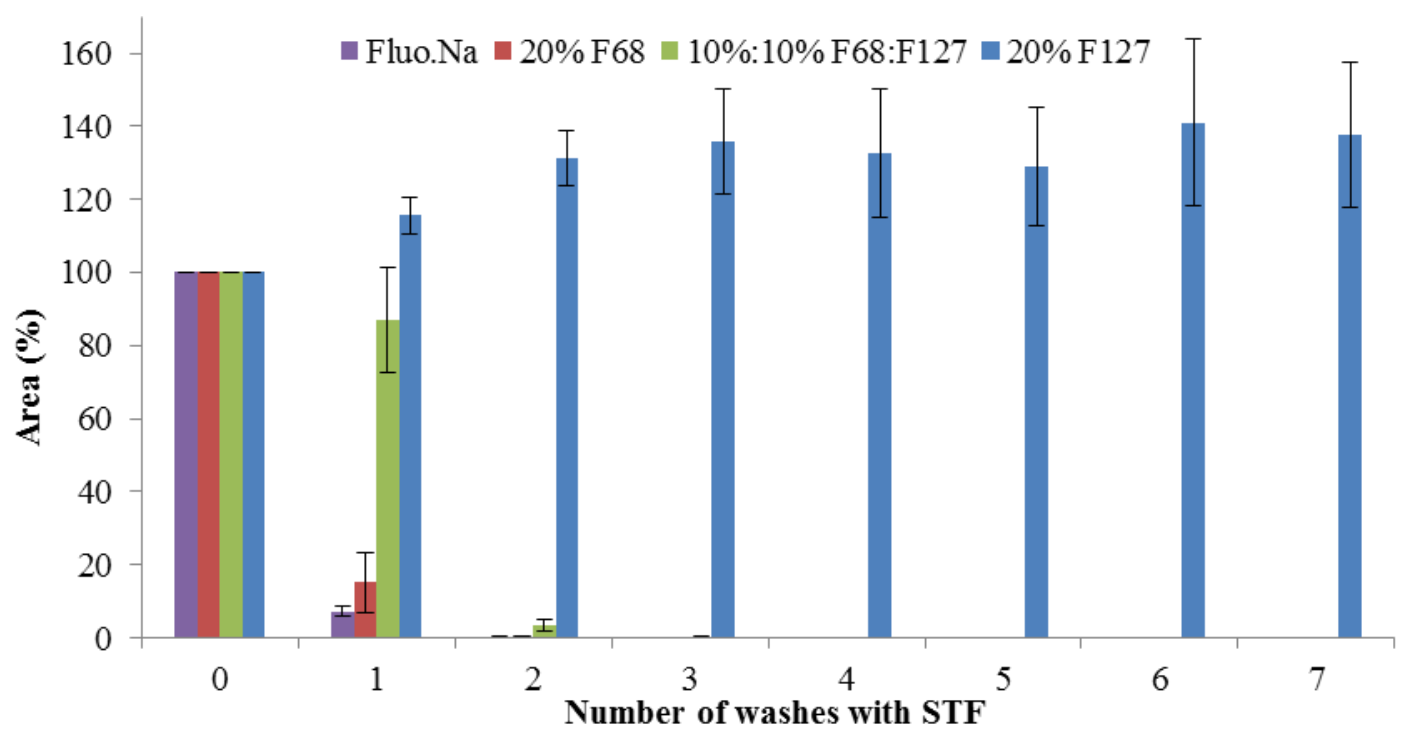


Figure 5. Retention of Pluronic formulations on glass surface $\left(37^{\circ} \mathrm{C}\right)$ after a number of consecutive washes (200 $\mu \mathrm{L} /$ wash) using simulated tear fluid (STF). (A) exemplary fluorescence images: a) Fluorescein sodium solution; b) $20 \mathrm{wt} \%$ F68 solution; c) $10 \mathrm{wt}$ $\%: 10 \%$ F68:F127 mixture; d) 20 wt \% F127 solution. Scale bar $=3$ mm. (B) retention profiles (mean \pm s.d., $n=3$ ). Each experiment was performed in triplicate with different eyes.

A

a

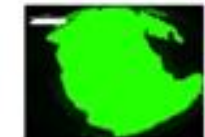

b

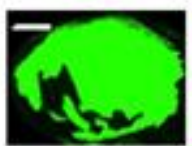

c

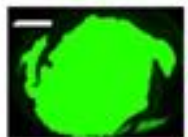

d

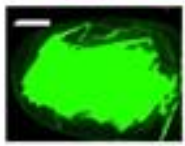

0
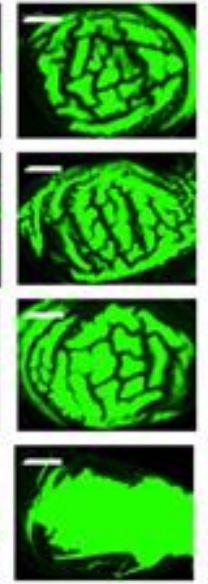

1
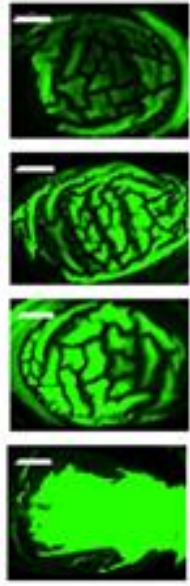

2
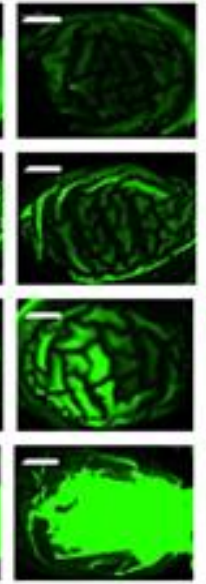

3
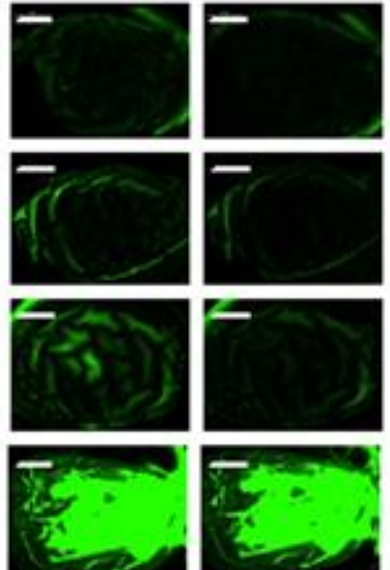

4

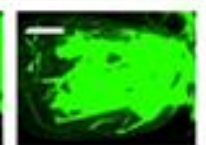

5

B

Number of washes with STF

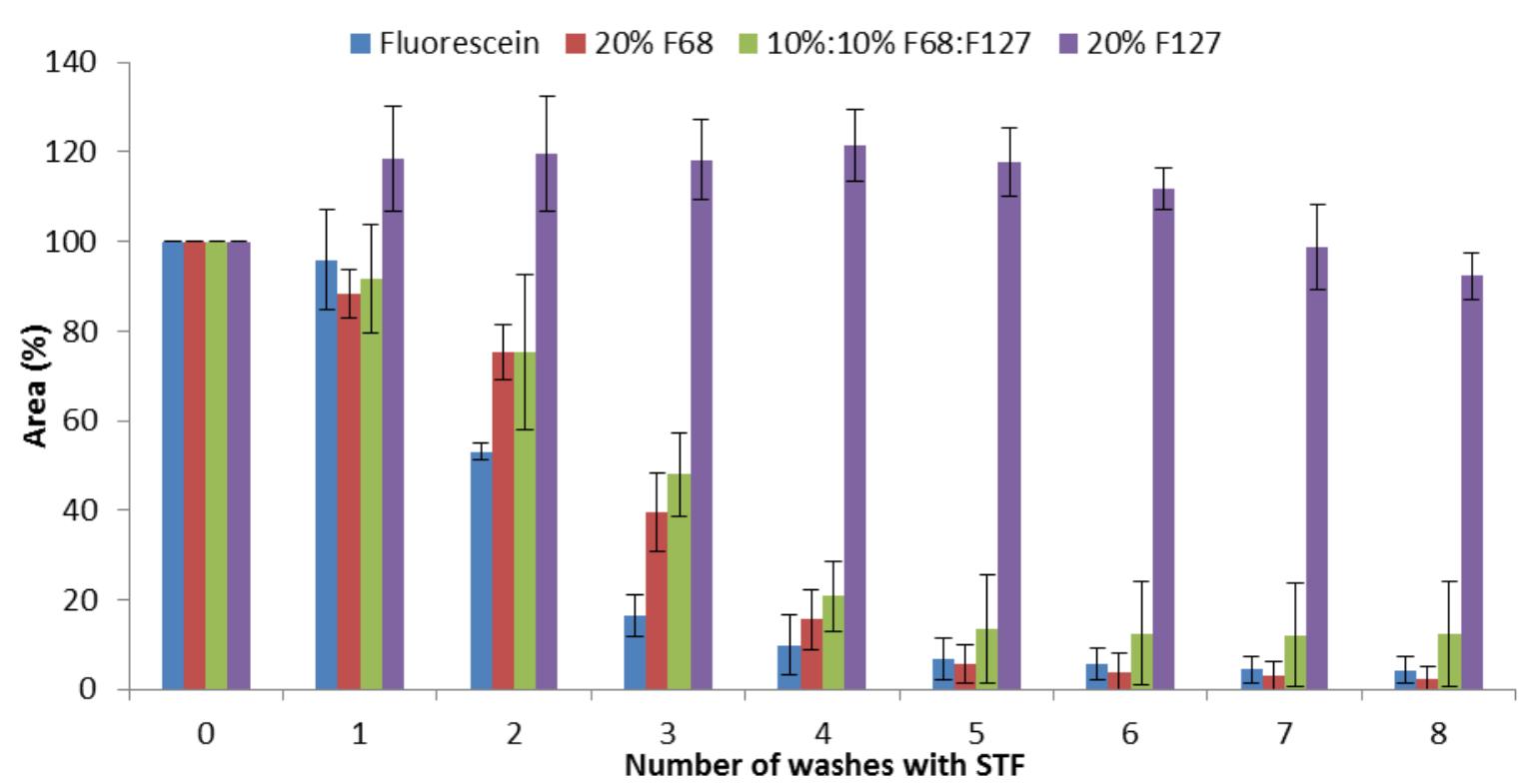

Figure 6. Retention of Pluronic formulations on freshly excised bovine cornea $\left(37^{\circ} \mathrm{C}\right)$ after a number of consecutive washes ( $200 \mu \mathrm{L} /$ wash) with simulated tear fluid (STF). (A) exemplary fluorescent images: a) Fluorescein sodium solution; b) $20 \mathrm{wt} \%$ F68 solution; c) 10\%:10\% F68:F127 mixture; d) 20 wt $\%$ F127 solution. Scale bar $=3 \mathrm{~mm}$ ). (B) retention profiles (mean \pm s.d., $\mathrm{n}=3$ ). Each experiment was performed in triplicate with different eyes. 


\subsection{In vivo retention of Pluronic formulations}

In vivo experiments were performed in rabbits $(\mathrm{n}=4)$ with $1 \mathrm{mg} / \mathrm{mL}$ solutions of fluorescein sodium either in $20 \mathrm{wt} \% \mathrm{~F} 127$ or $20 \mathrm{wt} \%$ F68. Only these two formulations were used in this study because our in vitro retention study showed that $20 \mathrm{wt} \% \mathrm{~F} 68$ had comparative retention with binary formulations. Each formulation was instilled into the rabbit's right eye and their retention was controlled both visually by taking photographs at regular time intervals and by taking regular swabs and analysing the presence of fluorescein sodium in the tear fluid using spectrophotometry. The formulations were specifically administered on the surface of the cornea rather than the lower cul-de-sac of the eye because of our interest in the development of drug delivery systems to the cornea. This targeting of the cornea is particularly important in some ocular conditions (e.g. keratoconus) (Morrison et al, 2013).

A
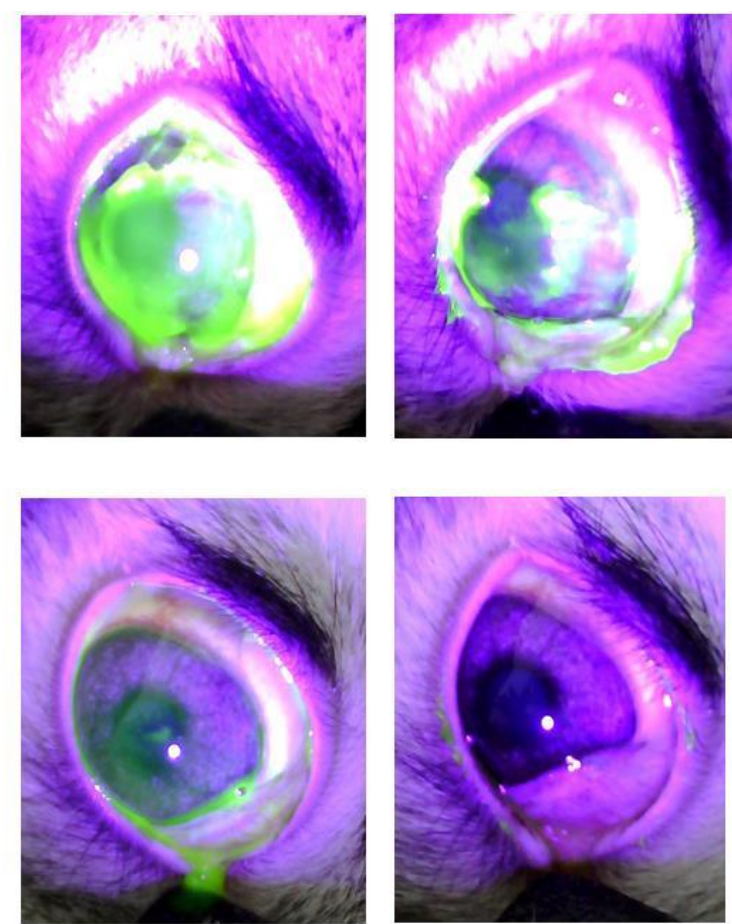

B

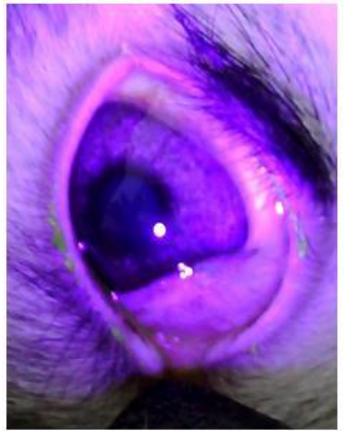

$5 \min$
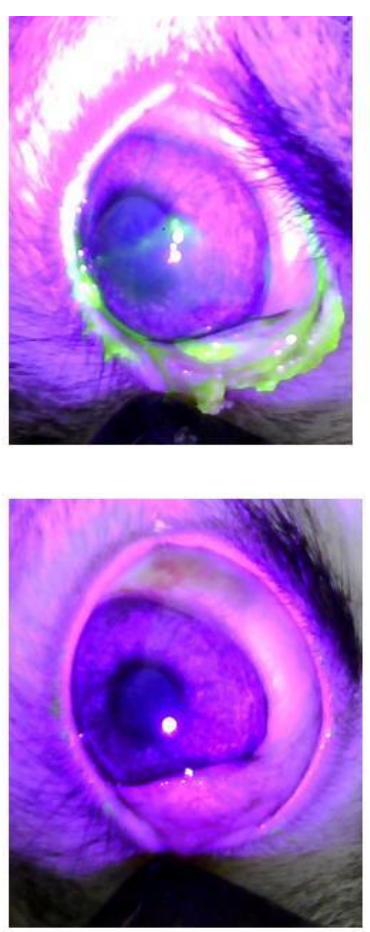

$20 \mathrm{~min}$
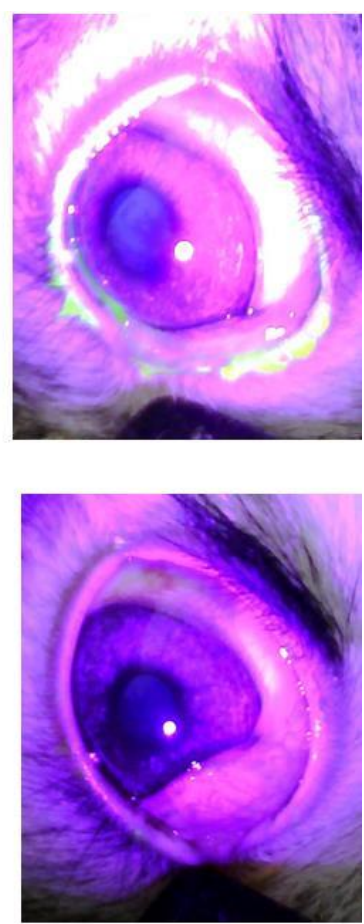

$30 \mathrm{~min}$ 


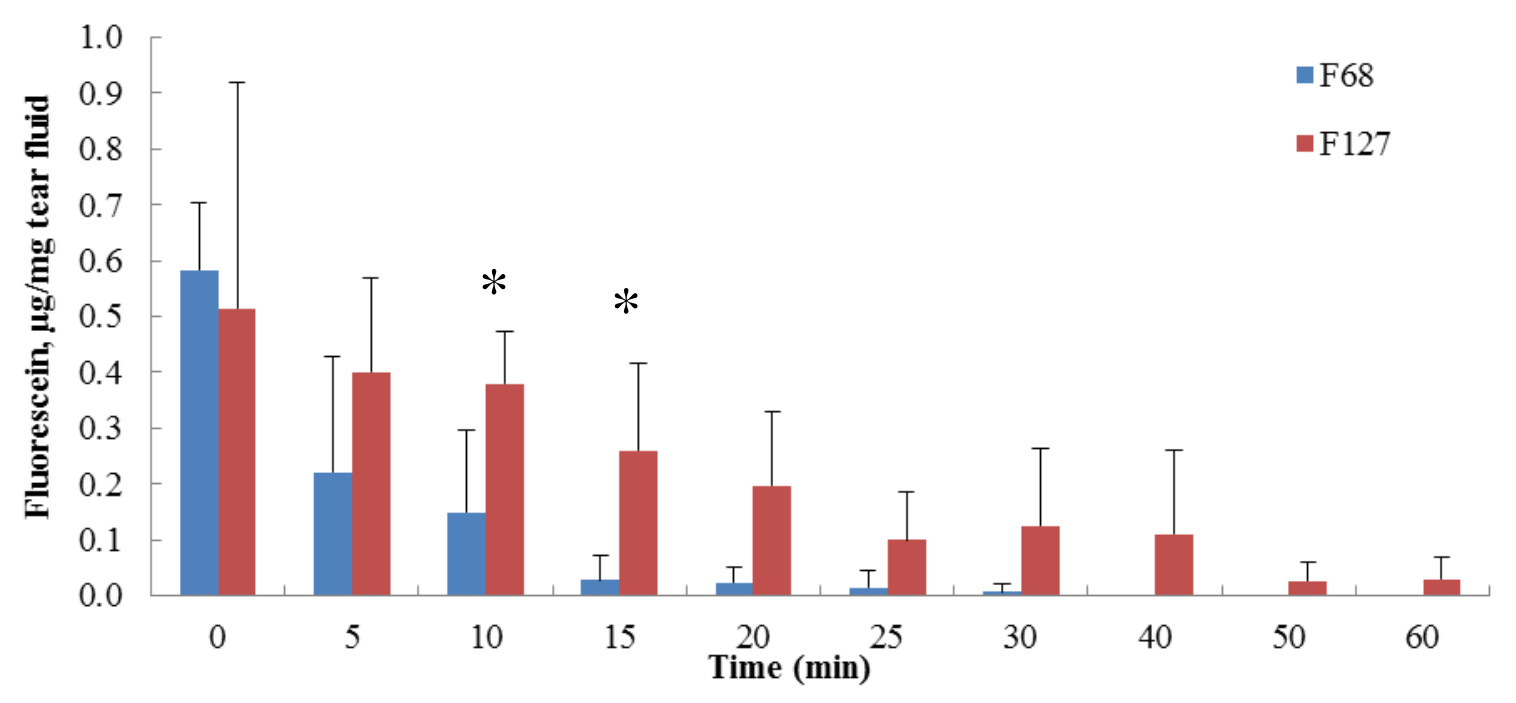

Figure 7. In vivo retention of Pluronic formulations containing $1 \mathrm{mg} / \mathrm{mL}$ fluorescein sodium solution. (A) exemplary images of rabbit eyes with $20 \mathrm{wt} \%$ F127 (a) and F68 (b) taken at different time intervals. Green colour shows the fluorescence from fluorescein sodium. (B) Content of fluorescein sodium in tear fluid as a function of time following ocular administration in vivo. (mean $\pm \mathrm{SD}, \mathrm{n}=4$, * represents $\mathrm{p}<0.05$ between groups).

Figure 7 shows exemplary images of rabbit eyes with Pluronic formulations administered in vivo and subsequent changes in the concentration of fluorescent sodium in the tear fluid. Administration of the F127 formulation resulted in immediate formation of a gel in situ, which provided even coverage of the cornea. However, unlike the in vitro experiments, this gel did not show very long residence and was washed off from the corneal surface within 10$15 \mathrm{~min}$. This substantial difference between the in vitro and in vivo results is likely related to the blinking reflex that ensured re-spreading of the formulation and its more efficient removal from the ocular surface. The formulation containing F68 did not show formation of the gel on the corneal surface and was almost immediately moved into the lower cul-de-sac of the eye upon first blinking. The analysis of the fluorescein sodium content in the tear fluid following in vivo administration indicated a significantly longer dye residence in the case of F127 formulation compared to F68. There was a significantly higher fluorescein concentration in the tear fluid at 10 and 15 min following administration of F127 formulation compared to F68 ( $\mathrm{p}<0.05$ ). No significant difference in fluorescein concentration in the tear fluid was observed after 20 min, indicating almost complete wash off in both cases (F127 and F68).

\section{Conclusion}

The thermal transition and gelation of aqueous Pluronic F127, Pluronic F68 and mixtures thereof have been studied using differential scanning calorimetry, rheological measurements and dynamic light scattering. The formation of a transparent gel under physiological conditions was observed only in the case of $20 \mathrm{wt} \%$ solutions of Pluronic F127. Pluronic F68 as well as its mixtures with Pluronic F127 formed gels only above the physiological 
temperature, which makes them unsuitable as thermally-responsive in situ gelling drug delivery systems. Aqueous solutions (20 wt \%) of Pluronic F127 and Pluronic F68 are nonirritant to mucosal tissues and therefore suitable for application in ocular drug delivery. In vitro and in vivo retention experiments conducted with Pluronic F127 and F68 formulations containing fluorescein sodium confirmed that aqueous Pluronic F127 formed gels upon ocular administration, which improved drug residence. The retention of non-gelling Pluronic F68 formulations was much weaker compared to Pluronic F127. 


\section{Acknowledgements}

We thank the Leverhulme Trust for funding the in vitro ocular retention experiments in this study (RPG-2013-017). The authors are grateful to Dr N. Aukenov and the team of undergraduate students of Semey State Medical University (V. Sarancha, A. Kisselyova, D. Bolatova, A. Bayakhmetov, D. Isakhanov, and R. Kutlubulatov) for technical assistance during in vivo experiments.

\section{References}

Adriaens, E., Remon, J.P. (1999). Gastropods as an evaluation tool for screening the irritating potency of absorption enhancers and drugs. Pharm. Res. 16 (8), 1240-1244.

Adriaens, E., Dhondt, M.M., Remon, J.P. (2005). Refinement of the Slug Mucosal Irritation test as an alternative screening test for eye irritation. Toxicol in Vitro. 19(1), 79-89.

Agrawal A.K, Das M., Jain S. (2012) In situ gel systems as 'smart' carriers for sustained ocular drug delivery. Expert Opin Drug Deliv. 9, 383-402.

Akash M.S., Rehman K. (2015) Recent progress in biomedical applications of Pluronic (PF127): Pharmaceutical perspectives. J. Control. Release. 209, 120-138.

Aseyev V., Tenhu H., Winnik F.M. (2011) Non-ionic thermoresponsive polymers in water. Adv. Polym. Sci. 242, 29-89.

Batrakova, E.V., Kabanov A.V. (2008) Pluronic Block Copolymers: Evolution of Drug Delivery Concept from Inert Nanocarriers to Biological Response Modifiers, J. Control. Release. 130(2): 98-106.

Beezer, A.E., Loh, W., Mitchell, J. C., Royall, P. G., Smith, D. O., Tute, M. S., Armstrong, J. K., Chowdhry, B. Z., Leharne, S. A., Eagland, D., Crowther, N. J. (1994). An Investigation of Dilute Aqueous Solutions Behaviour of Poly(oxyethylene) + Poly(oxypropylene) + Poly(oxyethylene) Block Copolymers. Langmuir. 10, 4001-4005.

Cave R.A., Cook J.P., Connon C.J., Khutoryanskiy V.V. (2012) A flow system for the online quantitative measurement of the retention of dosage forms on biological surfaces using spectroscopy and image analysis, Int. J. Pharm., 428, 96-102.

Chung, Y.-I., Lee, S.-Y., Tae, G. (2006). The effect of heparin on the gelation of Pluronic F127 hydrogel. Colloids \& Surfaces A: Physicochem. Eng. Aspects. 284-285, 480-484. 
Cho, K.Y., Chung, T.W., Kim, B.C., Kim, M.K., Lee, J.H., Wee, W.R., Cho, C.S. (2003). Release of ciprofloxacin from poloxamer-graft-hyaluronic acid hydrogels in vitro. Int. J. Pharm. 260 (1), 83-91.

Cook M.T., Smith S.L., Khutoryanskiy V.V. (2015) Novel glycopolymer hydrogels as mucosa-mimetic materials to reduce animal testing, Chem. Commun., 51, 14447-14450.

Edsman, K., Carlfors, J., Petersson, R. (1998). Rheological evaluation of poloxamer as an in situ gel for ophthalmic use. European Journal of Pharmaceutical Sciences. 6, 105-112.

El-Kamel A.H. (2002) In vitro and in vivo evaluation of Pluronic F127-based ocular delivery system for timolol maleate. Int. J. Pharm. 241, 47-55.

Hughes P.M., Olejnik O., Chang-Lin J.-E., Wilson C.G. (2005) Topical and systemic drug delivery to the posterior segments, Adv. Drug Delivery Reviews, 57, 2010-2032.

Ibrahim el-S.A., Ismail S., Fetih G., Shaaban O., Hassanein K., Abdellah N.H. (2012) Development and characterization of thermosensitive pluronic-based metronidazole in situ gelling formulations for vaginal application. Acta Pharm. 62, 59-70.

Irmukhametova G.S., Mun G.A., Khutoryanskiy V.V. (2011) Thiolated mucoadhesive and PEGylated non-mucoadhesive organosilica nanoparticles from 3-mercaptopropyltrimethoxysilane, Langmuir, 27, 9551-9556.

Järvinen K., Järvinen T., Urtti A. (1995) Ocular absorption following topical delivery, Adv. Drug Delivery Reviews, 16, 3-19.

Kadam Y., Yerramilli U., Bahadur A. (2009) Solubilization of poorly water-soluble drug carbamezapine in Pluronic ${ }^{\circledR}$ micelles: Effect of molecular characteristics, temperature and added salt on the solubilizing capacity, Colloids \& Surfaces B: Biointerfaces, 72, 141-147.

Khutoryanskaya, O. V., Mayeva, Z. A., Mun, G. A., Khutoryanskiy, V. V. (2008). Designing Temperature-Responsive Biocompatible Copolymers and Hydrogels Based on 2Hydroxyethyl(meth)acrylates. Biomacromolecules. 9, 3353-3361.

Khutoryanskaya O.V., Morrison P.W.J., Seilkhanov S.K., Mussin M.N., Ozhmukhametova E.K., Rakhypbekov T.K., Khutoryanskiy V.V. (2014) Hydrogen-bonded complexes and blends of poly(acrylic acid) and methylcellulose: nanoparticles and mucoadhesive films for ocular delivery of riboflavin, Macromol. Biosci., 14, 225-234.

Lin H.R., Sung K.C., Vong W.J. (2004) In situ gelling of alginate/pluronic solutions for ophthalmic delivery of pilocarpine. Biomacromolecules. 5, 2358-2365.

Ludwig A. (2005) The use of mucoadhesive polymers in ocular drug delivery, Adv. Drug Delivery Reviews, 57, 1595-1639. 
Morrison, P. W. J., Connon, C. J., Khutoryanskiy, V. V. (2013). Cyclodextrin-Mediated Enhancement of Riboflavin Solubility and Corneal Permeability. Molecular Pharmaceutics. 10 (2), 756-762.

Morrison P.W.J., Khutoryanskiy V.V. (2014). Advances in Ophthalmic Drug Delivery, Therapeutic Delivery, 5 (12), 1307-1325.

Mun, E. A., Morrison, P. W. J., Williams, A. C., Khutoryanskiy, V. V. (2014). On the Barrier Properties of the Cornea: A Microscopy Study of the Penetration of Fluorescently Labeled Nanoparticles, Polymers, and Sodium Fluorescein. Molecular Pharmaceutics. 11 (10), 35563564.

Nurkeeva Z.S., Mun G.A., Khutoryanskiy V.V., Bitekenova A.B., Dzhusupbekova A.B. (2002) Polymeric complexes of poly(acrylic acid) and poly(2-hydroxyethyl vinyl ether) with lidocaine hydrochloride, J. Biomat. Sci. Polym. Edn. 13, 759-768.

Nurkeeva Z.S., Khutoryanskiy V.V., Mun G.A., Sherbakova M.V., Ivaschenko A.T., Aitkhozhina N.A. (2004) Polycomplexes of poly(acrylic acid) with streptomycin sulfate and their antibacterial activity, Europ. J. Pharm. \& Biopharm., 57, 2, 245-249.

Oh, K. T., Bronich, T. K., Kabanov, A.V. (2004). Micellar formulations for drug delivery based on mixtures of hydrophobic and hydrophilic Pluronic block copolymers. J. Control. Release. 94 (1), 411-422.

Pandit N.K., McGowan R. (1998) Gelation of Pluronic ${ }^{\circledR}$ F127-Polyethylene Glycol Mixtures: Relationship to PEG Molecular Weight. Drug Devel. Ind. Pharm. 24, 183-186.

Sharma, P. K., Bhatia, S. R. (2004). Effect of anti-inflammatories on Pluronic ${ }^{\circ}$ F127: micellar assembly, gelation and partitioning. Int. J. Pharm. 278 (2), 361-377.

Sek L., Boyd B.J., Charman W.N., Porter C.J. (2006) Examination of the impact of a range of Pluronic surfactants on the in-vitro solubilisation behaviour and oral bioavailability of lipidic formulations of atovaquone. J. Pharm. Pharmacol. 58(6), 809-820.

Thrimawithana T.R., Rupenthal I.D., Young S.A., Alany R.G. (2012) Environment-sensitive polymers for ophthalmic drug delivery. J. Drug Delivery Science and Technology 22, 117124.

Varshosaz J., Tabbakhian M., Salmani Z. (2008) Designing of a thermosensitive Chitosan/Poloxamer in situ gel for ocular delivery of ciprofloxacin. The Open Drug Delivery Journal, 2, 61-70.

Wei, G., Xu, H., Ding, P. T., Li, S. M., Zheng, J. M. (2002). Thermosetting gels with modulated gelation temperature for ophthalmic use: the rheological and gamma scintigraphic studies. J. Control. Release. 83 (1), 65-74.

Withers C.A., Cook M.T., Methven L., Gosney M.A., Khutoryanskiy V.V. (2013) Investigation of milk proteins binding to the oral mucosa, Food \& Function, 4, 1668-1674. 
Zhang, M., Djabourov, M., Bourgaux, C., Bouchemal, K. (2013). Nanostructured fluids from pluronic ${ }^{\circledR}$ mixtures. Int. J. Pharm. 454, 599-610. 


\section{Supporting Information}

Table S1. Composition of F68:F127 binary systems. Pluronic flakes and the vehicles were stirred at $4{ }^{\circ} \mathrm{C}$ until the polymers were totally dissolved. Vehicles used included deionised water, STF, and fluorescein sodium salt $(1 \mathrm{mg} / \mathrm{mL})$ in STF stock solution. Vehicles used depending on the experiment set up.

\begin{tabular}{|c|c|c|}
\hline $\begin{array}{c}\text { F68:F127 binary system } \\
(\% \mathrm{w} / \mathrm{v})\end{array}$ & F68 $(\mathrm{g})$ & F127 $(\mathrm{g})$ \\
\hline $0: 20$ & 0 & 2.0 \\
\hline $2: 18$ & 0.2 & 1.8 \\
\hline $4: 16$ & 0.4 & 1.6 \\
\hline $6: 14$ & 0.6 & 1.4 \\
\hline $8: 12$ & 8.0 & 1.2 \\
\hline $10: 10$ & 10.0 & 10.0 \\
\hline $20: 0$ & 20.0 & 0 \\
\hline
\end{tabular}

Table S1 details the amount of Pluronics in each formulation. Samples differred mainly in the solvents used and the presence of other solutes.

Deionised water was used for micellisation tests. For gelation experiment, two sets were prepared with solvents including deionised water and STF. While for the erosion test, Pluronics were dissolved in STF. In retention test, Pluronics were dissolved in a stock solution of $1 \mathrm{mg} / \mathrm{mL}$ fluorescein sodium salt in STF. 


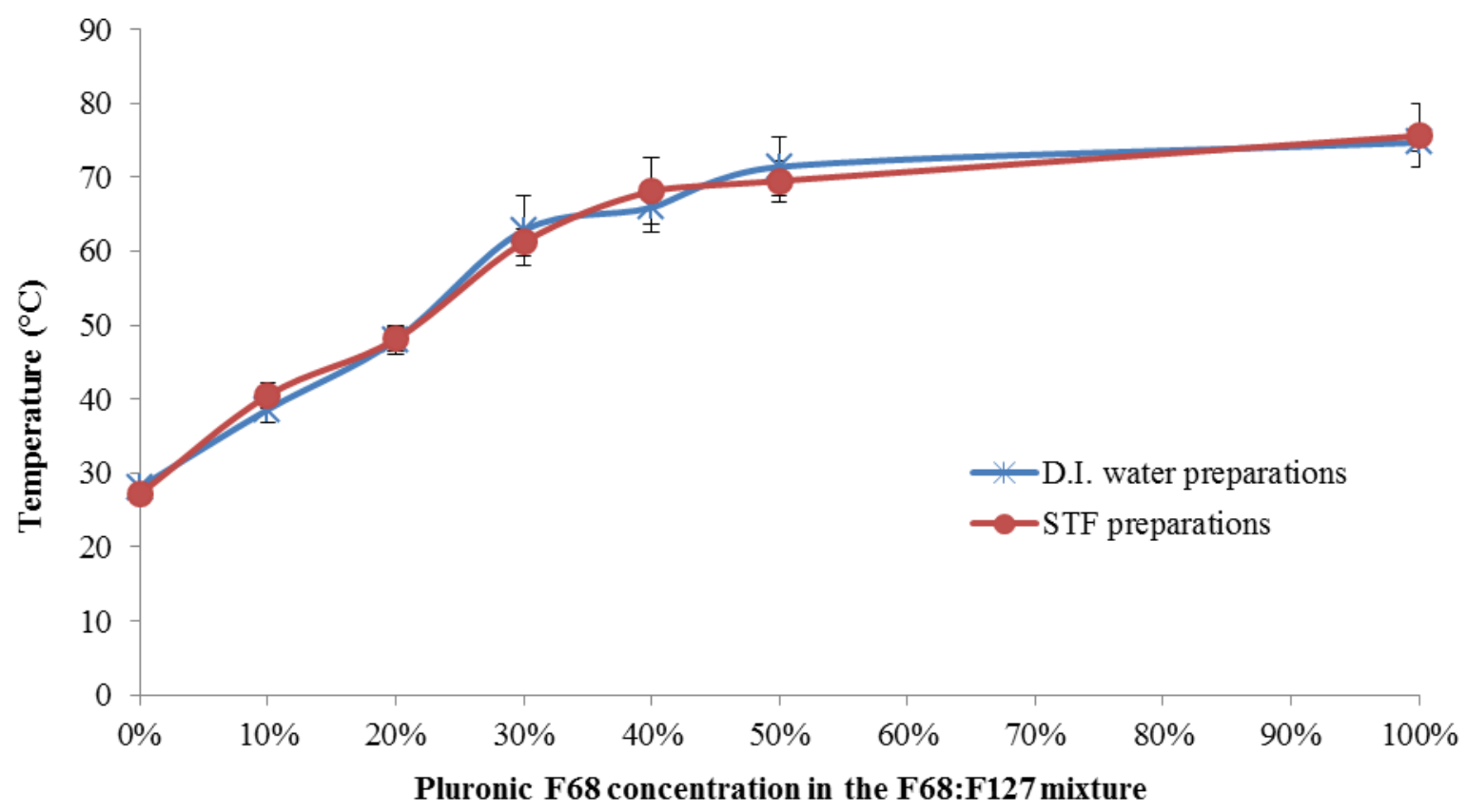

Figure S1. Changes in gelation temperature (Tg) of different F68:F127 binary systems, where 0\% F68 represents the 20\% (w/v) F127 sample, while 100\% F68 represents the 20\% (w/v) F68 sample (mean \pm s.d., $n=3)$.

Figure $\mathrm{S} 1$ represents the changes in gelation temperature of the Pluronics binary systems prepared using deionised water and STF with ofloxacin. The acquired data showed similar gelation patterns regardless of the utilised vehicles. Formulations were kept at least 48 hours at $4{ }^{\circ} \mathrm{C}$ before carrying out the experiment in order to reduce the shear history of the tested samples. 

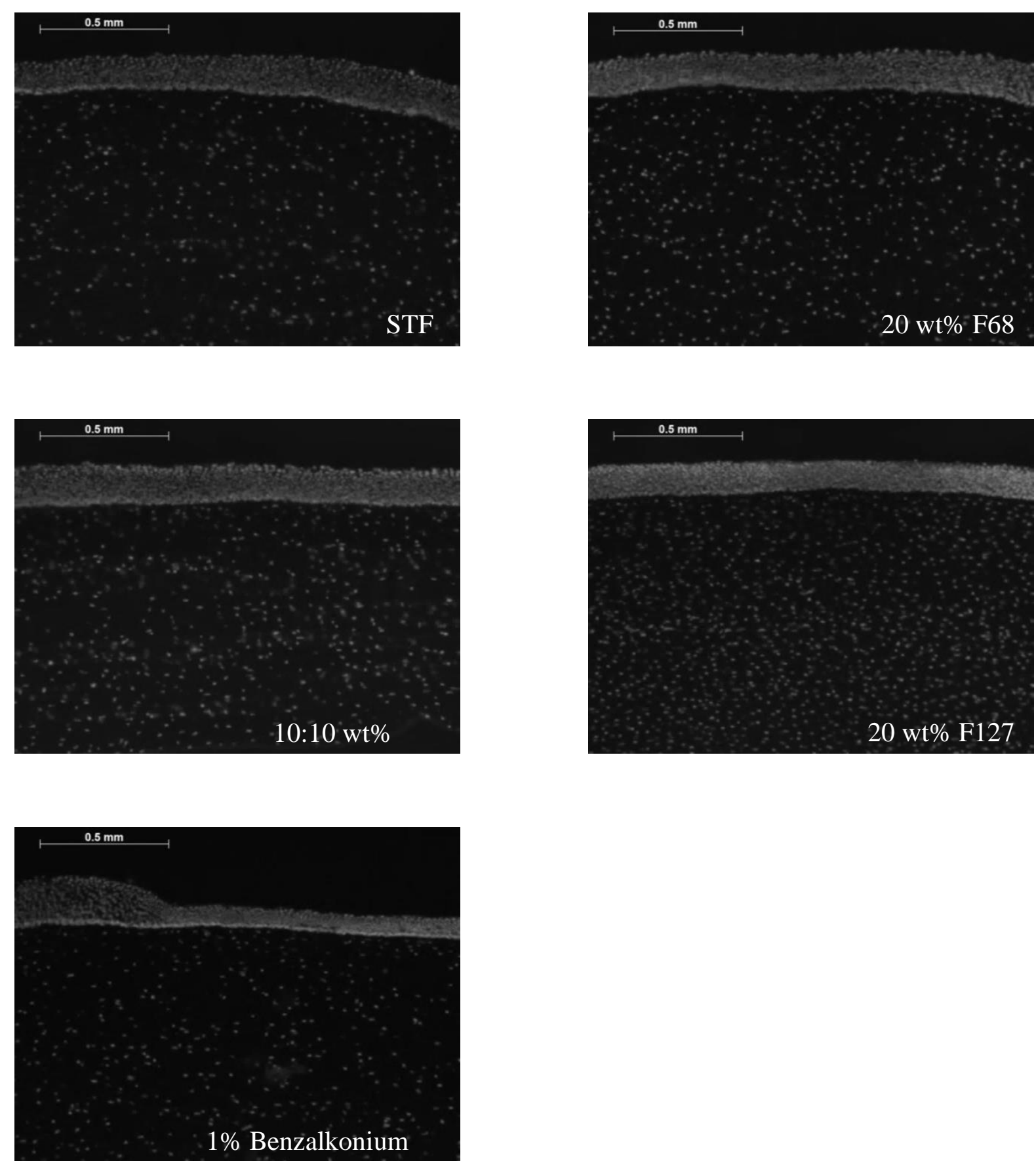

Figure S2. Effect of Pluronics on corneal epithelium

Figure S2 illustrates the effect of Pluronics, their mixtures and control solutions on the epithelial layer of bovine cornea ex vivo. Each picture shows the area covered by the tested sample (within the donor part of Franz cell) and the area not affected by it. Simulated tear fluid was used as a negative control and $1 \%$ benzalkonium chloride as a positive control. 
It can be noticed that $1 \%$ benzalkonium chloride sample caused considerable damage to the exposed corneal epithelium compared to the rest of cornea, while the other systems had no such effect on epithelial thickness. Size bar is $0.5 \mathrm{~mm}$. 